\title{
Early formation pathways of surfactant micelle directed ultrasmall silica ring and cage structures
}

\author{
Kai Ma ${ }^{1 \dagger}$, Katherine A. Spoth ${ }^{2}$, Ying Cong ${ }^{1}$, Duhan Zhang ${ }^{1}$, Tangi Aubert ${ }^{1,3}$, Melik Z. Turker ${ }^{1}$, Lena F. \\ Kourkoutis ${ }^{2,4}$, Eduardo Mendes ${ }^{5}$ and Ulrich Wiesner ${ }^{1 *}$ \\ ${ }^{1}$ Materials Science Engineering, Cornell University, Ithaca, NY 14853, USA \\ ${ }^{2}$ Applied Physics, Cornell University, Ithaca, NY 14853, USA \\ ${ }^{3}$ Department of Chemistry, Ghent University, Ghent 9000, Belgium \\ ${ }^{4}$ Kavli Institute at Cornell for Nanoscale Science, Ithaca, NY 14853, USA \\ ${ }^{5}$ Advanced Soft Matter, Department of Chemical Engineering, Delft University of Technology, Van der Maasweg 9, 2629 \\ HZ Delft, The Netherlands. \\ *Correspondence to: ubw1@ cornell.edu
}

\section{Supporting Information Placeholder}

\begin{abstract}
By combining a surfactant, an organic pore expander, a silane, and poly(ethylene glycol) (PEG), we have observed the formation of a previously unknown set of ultrasmall silica structures in aqueous solutions. At appropriate concentrations of reagents, $\sim 2 \mathrm{~nm}$ primary silica clusters arrange around surfactant micelles to form ultrasmall silica rings, which can further evolve into cage-like structures. With increasing concentration, these rings line up into segmented worm-like one-dimensional (1D) structures, an effect that can be dramatically enhanced by PEG addition. PEG adsorbed 1D striped cylinders further arrange into higher order assemblies in the form of two-dimensional (2D) sheets or three-dimensional (3D) helical structures. Results provide insights into synergies between deformable noncovalent organic molecule assemblies and covalent inorganic network formation as well as early transformation pathways from spherical soft materials into 1D, 2D, and 3D silica solution structures, hallmarks of mesoporous silica materials formation. The ultrasmall silica ring and cage structures may prove useful in nanomedicine and other nanotechnology based applications.
\end{abstract}

Ultrasmall inorganic particles with sizes in the single-nanometer regime have recently attracted significant research attention due to their unique size-dependent properties e.g. in optics, catalysis, and medicine. ${ }^{1-3}$ Recent advances in electron microscopy have provided new mechanistic insights into their formation enabling unprecedented synthesis control. ${ }^{4-6}$ Ultrasmall inorganic particles have therefore become one of the most promising building blocks for bottom-up fabrication of novel nanomaterials ${ }^{7-9}$ and superlattices. ${ }^{10-12}$ For crystalline nanomaterials, geometrical anisotropy is an important driver of structure control in self-assembly. ${ }^{13,14}$ Amorphous inorganic particles are lacking this control parameter and organic templates or scaffolds are typically employed to direct structure. ${ }^{15,16}$ Examples include surfactant self-assembly directed silica formation, which since first discoveries were reported ${ }^{17,18}$ has seen steady progress in morphological control. ${ }^{19,20}$

Despite growing appreciation that clusters about $2 \mathrm{~nm}$ in size are the primary building blocks of various templated silica nano- and mesostructures, ${ }^{21}$ elucidation of early formation and structural transformation pathways and their complexity has remained limited. This is due in part to the dynamic nature of hybrid materials formation, which simultaneously involves noncovalent assembly, e.g. of surfactants into micelles and their arrangement into lattices, as well as covalent linking of structural units, e.g. the condensation of primary silica particles via Si-O-Si bond formation. While progress has recently been made on the organic/polymeric materials side, ${ }^{22-24}$ detailed understanding of the pathway complexity of simultaneously occurring noncovalent and covalent assembly processes that synergistically influence each other is far less developed in organic-inorganic hybrid materials. In particular, interest in early formation pathways of nanoscopic silica ${ }^{25}$ has recently been renewed with the discovery of surfactant micelle directed ultrasmall silica cages with highly symmetric dodecahedron structure. ${ }^{26}$

Here, we report on transmission electron microscopy (TEM) and cryo-state TEM (cryo-EM) investigations of early aqueous solution structures of a surfactant (cetrimonium bromide, CTAB), a pore expander (mesitylene, TMB), a silane (tetra methyl/ethyl orthosilicate, TMOS/TEOS), and poly(ethylene glycol) (PEG) at different concentrations (Fig. 1a). When adding TMOS into aqueous solutions of TMB-swollen CTAB micelles at pH around 8 (Fig. $1 \mathrm{~b}$ and c), negatively-charged primary silica clusters $\sim 2 \mathrm{~nm}$ in size were observed (inset Fig. 1e) that associate with positively-charged micelle surfaces into ring-like geometries (Fig. 1d and e). Ring structures were corroborated by analysis of a TEM tilt series (Fig. S1). Cryo-EM images prepared using the native reaction solution showed projections of rings oriented randomly in three-dimensional space with $\sim 10 \mathrm{~nm}$ sized stripes representing ring top views (Fig. 1d and Fig. S2a). In comparison, TEM images showed the side view of the rings, where individual primary silica clusters in the ring-like assemblies could be identified (Fig. 1e and Fig. S2b). These clusters were further corroborated in experiments with assemblies covalently PEGylated using silane-functionalized PEGs (PEG-silane) based protocols. ${ }^{27}$ After surfactant template removal from PEGylated assemblies, TEM showed ultrasmall PEGylated silica particles with average hydrodynamic size from dynamic light scattering (DLS) around 4nm (Fig. S2, d and q), indicating a reversible assembly mechanism. 


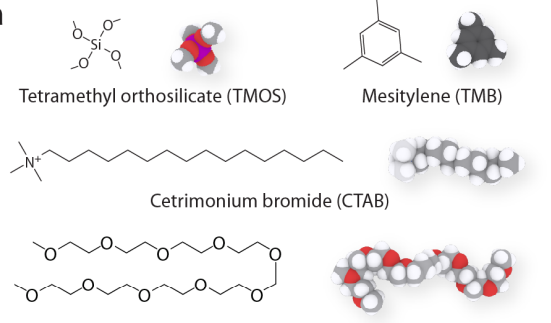

poly(ethylene glycol) (PEG)

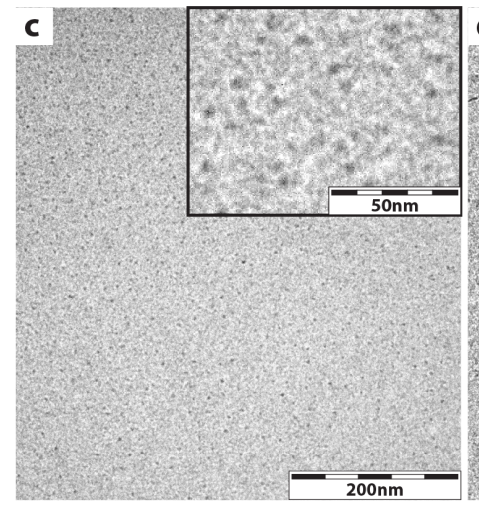

b

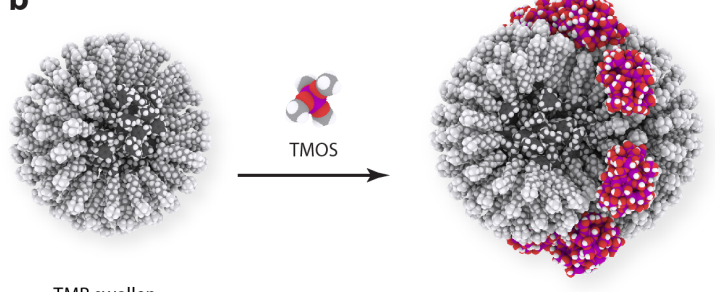

TMB swollen

CTAB micelle
Silica ring
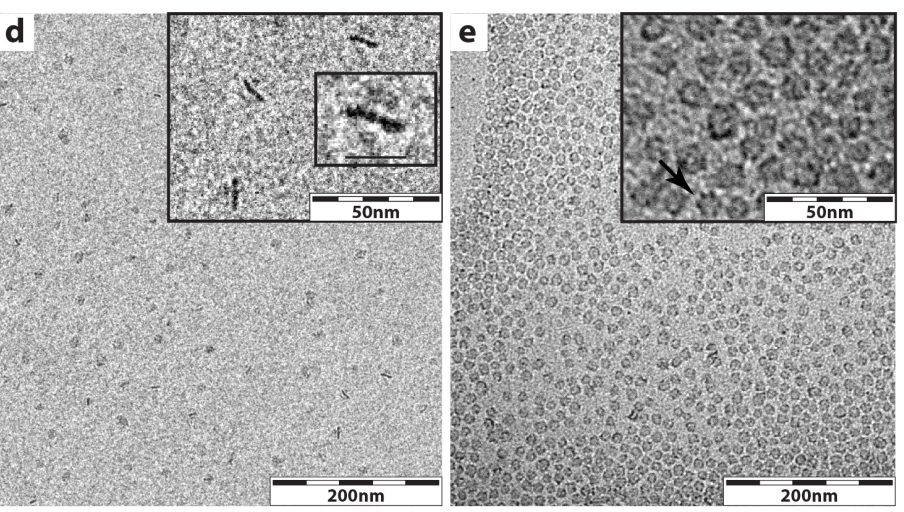

Fig. 1. Synthesis and characterization of silica rings. a, Chemical structures. b, Molecular graphic of the formation of silica rings. c, CryoEM image of TMB swollen CTAB micelles. d and e, Cryo-EM (d) and TEM (e) images of silica rings assembling on micelle surface. Insets in $\mathbf{c}$ to $\mathbf{e}$ display the zoomed-in Cryo-EM and TEM images, where the black arrow in the inset of $\mathbf{e}$ indicates the $\sim 2 \mathrm{~nm}$ primary silica clusters on micelle surface. In the molecular renderings, silicon, oxygen, carbon, and hydrogen are colored in purple, red, gray, and white, respectively. Different gray scales are applied to the carbon atoms of TMB and of heads and tails of CTAB for better visualization. Scale bar in the inset in $\mathbf{d}$ is $10 \mathrm{~nm}$.

Considering that no approach for symmetry breaking, e.g. electrostatic or magnetic fields, ${ }^{28,29}$ was applied in the synthesis, the fact that the primary silica clusters self-assemble into a ring-like geometry on the micelle surface is surprising. This assembly-based symmetry breaking is different from previously reported mesoporous silica particles with similar size, ${ }^{30}$ where pore expanding molecules (e.g. TMB) were absent. Therefore, we speculate that the ring formation is due to increased deformability of TMB swollen micelles as compared to TMB-free micelles (Fig. 1b).

Upon association of primary particles, the micelle surface is expected to deform, partially wrapping around the clusters due to electrostatic attraction between silica and CTAB head groups (Fig. 2a). However, this micelle surface deformation is unfavorable in terms of the Gibbs free energy of a spherical micelle. Lining up clusters in the form of a ring is expected to minimize the overall assembly Gibbs free energy relative to arbitrary cluster positions (Fig. 2a) by sharing dented micelle surfaces and removing deformations along the ring direction. This hypothesis is corroborated by a simple model calculation described in the Supporting Information (Fig. S3).

This proposed mechanism is also consistent with the quantitative analysis of the silica rings (Fig. 2b). The thickness of the rings is about $1.6 \mathrm{~nm}$, consistent with the size of the primary silica clusters. The inner and outer diameters of the rings are about $6.2 \mathrm{~nm}$ and $10.2 \mathrm{~nm}$, respectively, with relatively wide size distributions, consistent with the size of TMB swollen CTAB micelles. ${ }^{31}$

Upon the formation of rings at the micelle equator, an increase in curvature of micelle poles can be expected (Fig. 2a). This change in turn may favor micelle fusion via the poles, leading to 1D assemblies (Fig. 2a). To facilitate this effect, we increased the con- centration of ring-micelle assemblies by doubling the concentrations of TMOS, CTAB, and TMB (green column in Fig. S2q). As a result, elongated worm-like structures were observed consisting of several silica rings with well-defined spacing (Fig. 3a and d).

a

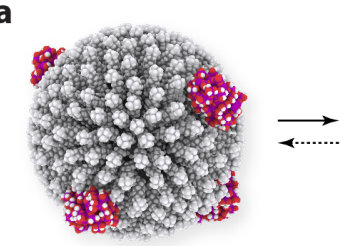

Random silica cluster distribution

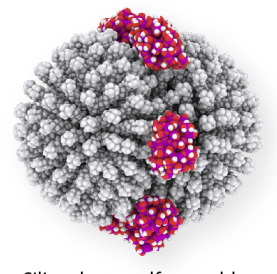

Silica cluster self-assembly b

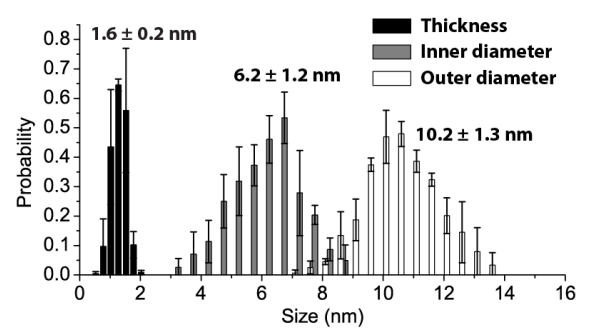

Fig. 2. Proposed Mechanism of silica ring formation. a, Comparison of different assembly approaches of silica clusters on micelle surface. The silica clusters could randomly distribute on the micelle surface (left), but rather assemble into a ring sharing the dented micelle surface (right). b, Dimensional analysis of silica rings. 

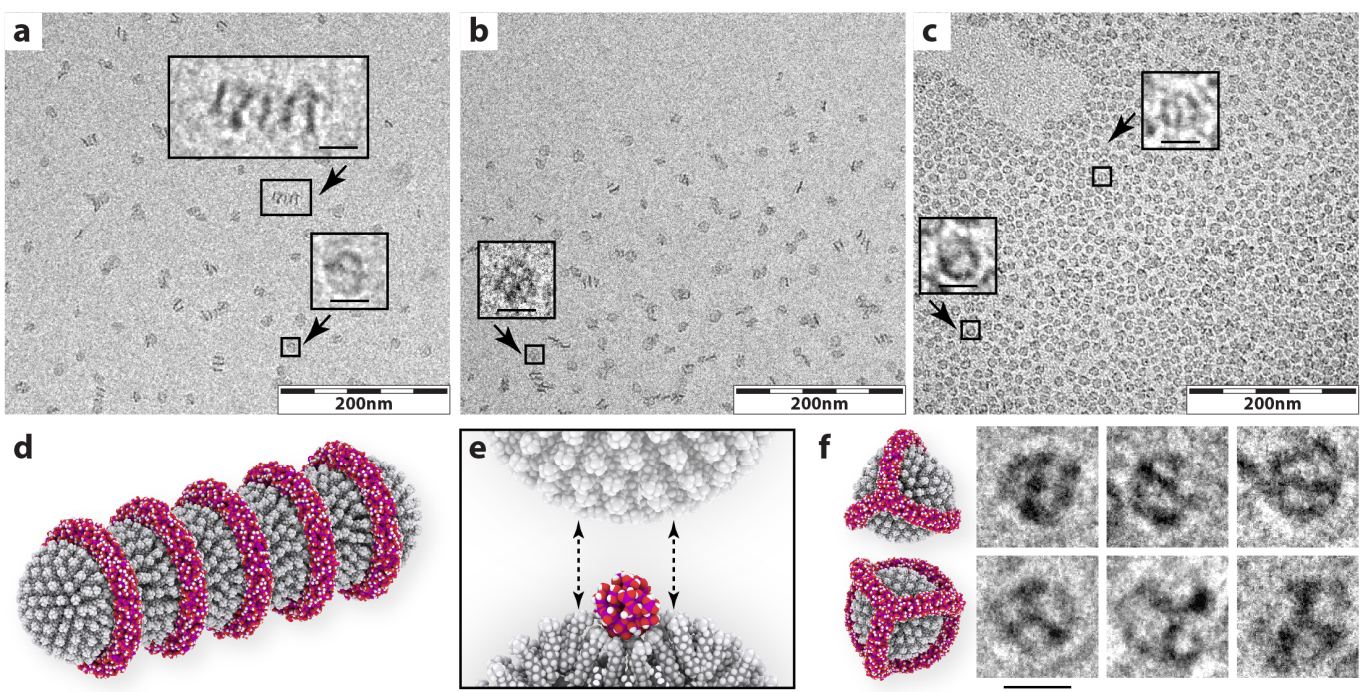

Fig. 3. 1D assembly of silica rings and different ring-like structures. a to c, Cryo-EM (a and b) and TEM (c) images of silica rings produced at the higher reaction concentration, i.e. the reaction conduction indicated in the green column in Fig. S2q. d, A molecular graphic of the worm-like 1D assembly of silica rings. e, Illustration of micelle surface wrapping around a silica cluster preventing attachment to other micelles (transparent top) due the strong electrostatic repulsion. f, Different structures of silica rings with more complex geometries, including nanoring with one additional arm (top raw) and tetrahedral cage (bottom raw).

After covalent PEGylation and removal of CTAB and TMB, structures now disassembled into narrowly dispersed PEGylated silica rings rather than ultrasmall silica particles (Fig. S2h) with average hydrodynamic size around $7 \mathrm{~nm}$ (Fig. S2q).

This $1 \mathrm{D}$ growth is very different from the conventional radial growth of mesoporous silica particles, where additional micelles attach to the growing primary particles thereby first leading to multi-arm star type structures and finally to multi-pore particles with spherical shape. ${ }^{30,32}$ In the formation of the silica rings, indenting the micelle surface seems to make approaches of other micelles to the opposite silica cluster side less likely because of increased electrostatic repulsion thereby suppressing templated radial particle growth, as illustrated in Fig. 3e.

It is interesting to note that in addition to silica rings, some cagelike assemblies with more complex structures were observed (Fig. 3 a to c). For example, some rings exhibited an additional arm (Fig. 3f, top), while symmetrical tetrahedral ultrasmall silica cages were also sometimes observed (Fig. 3f bottom). These higher-order structures point to a connection between the rings and the recently reported dodecahedral ultrasmall silica cages ${ }^{26}$ where the ring likely is the simplest in the family of assembly structures of primary silica clusters on the surface of deformable micelles.

The dimensions of the silica rings and their 1D assemblies can be tuned by dosing additional TEOS after primary ring formation. ${ }^{25}$ As compared to TMOS, TEOS has a slower hydrolysis rate suppressing secondary nucleation and facilitating direct condensation onto the existing silica rings. ${ }^{25}$ Starting with a small amount of TEOS (orange column in Fig. S2q), the thickness of the silica rings remained almost unchanged (Fig. S2K and Fig. S4), but TEM images of covalently PEGylated and cleaned samples showed narrowly size dispersed silica rings with reduced contrast variations (Fig. S2I) as compared to those formed solely from primary clusters (Fig. 1d and e). This was likely the result of additional silane mon- omers primarily condensing into the interstitial space left by aggregation of primary silica clusters (Fig. 1d and e). When the amount of dosed TEOS was further increased (purple column in Fig. S2q), silica rings grew thicker and the ring-to-ring distance in 1D assemblies increased (Fig. S4). In contrast, the interstitial spacing between adjacent rings, obtained by subtracting the ring thickness from the ring-to-ring distance (Fig. S4), remained unchanged at around $3.3 \mathrm{~nm}$. These data revealed that the interstitial spacing in the 1D structures was independent from the silica dimensions, likely the result of electrostatic repulsion between the silica rings (Fig. 3a and d).

Adding unfunctionalized PEG (i.e. without silane functionalization) into the reaction solution (black column in Fig. S2q) dramatically facilitated 1D ring assembly. Upon PEG addition, isolated rings (Fig. 1d and e) lined up into extended worm-like and striped 1D structures (Fig. 4a). Each of the 1D assemblies was composed of tens of silica rings. Similar elongated worm-like striped cylinders were observed when PEGs with decreased chain length and different end-groups were added (Fig. S5). These short PEG molecules had minor effects on solution viscosity, nor were they long enough to directly bridge between two adjacent silica rings in the1D assemblies. Rather, the formation of the elongated 1D assemblies from PEG addition was likely due to the increased stability of ring assemblies caused by hydrogen bonding between PEGs and silica as well as among PEGs on different rings mediated by hydrogen bonding with water (Fig. S5d). ${ }^{27}$

The adherence of PEGs to silica also facilitated self-assembly of the 1D striped cylinders into more complex 2D and 3D structures. Through drying the reaction solution on a TEM substrate, 2D hierarchical arrays of silica rings were generated via the side-by-side packing of PEG adsorbed worm-like 1D assemblies (Fig. 4b). Furthermore, 3D structures consisting of assemblies of two or more worm-like 1D structures into what appeared to be helical 

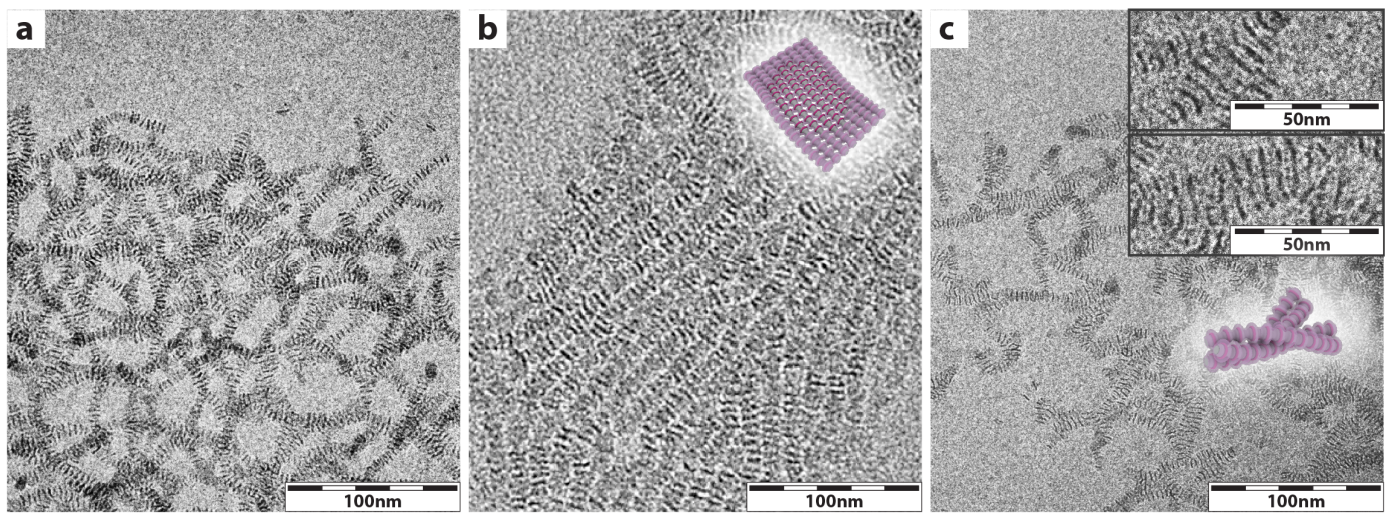

Fig. 4. 1D, 2D, and 3D ring assemblies facilitated by PEGs. a, Cryo-EM images of elongated and segmented 1D ring assemblies obtained upon PEG addition. Reaction conditions are indicated by the black column in Fig. S2q. TEM images of corresponding assemblies without PEG addition are shown in Fig. 1d and e. b, TEM images of 2D hierarchical arrays of silica rings obtained through drying of solutions of PEG-addition based 1D ring assemblies on a planar TEM substrate. c, Cryo-EM images of 3D assemblies of 1D ring assemblies facilitated by PEGs.

configurations were observed in cryo-state TEM (Fig. 4c). Most of the silica rings aligned next to each other in these 2D and 3D structures (Fig. $4 \mathrm{~b}$ and c). This is consistent with PEGs bridging the silica rings of different striped cylinders thereby promoting complex assembly.

The observed formation of rings and cages, as well as transformations from individual rings to $1 \mathrm{D}, 2 \mathrm{D}$ and $3 \mathrm{D}$ hierarchical assemblies, may provide mechanistic insights to the formation of structural building blocks, as well as to early morphological transformations, in well-known surfactant templated mesoporous materials. ${ }^{18,26,33,34}$ It may further enable the synthesis of hitherto unknown silica nanostructures. The thickness of the rings is less than $2 \mathrm{~nm}$ with around 4 to 5 tetrahedral $\mathrm{SiO}_{2}$ units across (Fig. 1b). PEGylated silica rings and cages are therefore expected to behave more like circular polymer chains with structural flexibility and may find applications as novel chemotherapy delivery vehicles leaving the body via efficient renal clearance pathways. ${ }^{3}$ Furthermore, since a recent study on silica cages with dedecahedral structure demonstrated that similar structures could also be obtained with metals and transition metal oxides, ${ }^{26}$ this work opens a wide field for novel ultrasmall nanomaterials discovery.

\section{REFERENCES}

1. P. D. Jadzinsky, G. Calero, C. J. Ackerson, D. A. Bushnell, R. D. Kornberg, Structure of a Thiol Monolayer-Protected Gold Nanoparticle at 1.1 A Resolution, Science 318, 430-433 (2007).

2. M. Turner, V. B. Golovko, O. P. H. Vaughan, P. Abdulkin, A. Berenguer-Murcia, M. S. Tikhov, B. F. G. Johnson, R. M. Lambert, Selective Oxidation with Dioxygen by Gold Nanoparticle Catalysts Derived from 55-Atom Clusters, Nature 454, 981-983 (2008).

3. E. Phillips, O. Penate-Medina, P. B. Zanzonico, R. D. Carvajal, P. Mohan, Y. Ye, J. Humm, M. Gönen, H. Kaliagian, H. Schöder, H. W. Strauss, S. M. Larson, U. Wiesner, M. S. Bradbury, Clinical Translation of an Ultrasmall Inorganic Optical-PET Imaging Nanoparticle Probe, Sci. Transl. Med. 6, 260ra149 (2014).

4. H. Zheng, R. K. Smith, Y.-W. Jun, C. Kisielowski, U. Dahmen, A. P. Alivisatos, Observation of Single Colloidal Platinum Nanocrystal Growth Trajectories, Science 324, 1309-1312 (2009).
5. J. M. Yuk, J. Park, P. Ercius, K. Kim, D. J. Hellebusch, M. F. Crommie, J. Y. Lee, A. Zettl, A. P. Alivisatos, High-Resolution EM of Colloidal Nanocrystal Growth Using Graphene Liquid Cells, Science 336, 61-64 (2012).

6. E. M. Pouget, P. H. H. Bomans, J. A. C. M. Goos, P. M. Frederik, G. D. With, N. A. J. M. Sommerdijk, The Initial Stages of TemplateControlled CaCO3 Formation Revealed by Cryo-TEM, Science 323, 1455-1458 (2009).

7. M. Templin, A. Franck, A. Du Chesne, H. Leist, Y. Zhang, R. Ulrich, V. Schädler, U. Wiesner, Organically Modified Aluminosilicate Mesostructures from Block Copolymer Phases, Science 278, 17951798 (1997).

8. S. C. Warren, L. C. Messina, L. S. Slaughter, M. Kamperman, Q Zhou, S. M. Gruner, F. J. DiSalvo, U. Wiesner, Ordered Mesoporous Materials from Metal Nanoparticle-Block Copolymer Self-Assembly, Science 320, 1748-1752 (2008).

9. C. Schliehe, B. H. Juarez, M. Pelletier, S. Jander, D. Greshnykh, M. Nagel, A. Meyer, S. Foerster, A. Kornowski, C. Klinke, H. Weller, Ultrathin PbS Sheets by Two-Dimensional Oriented Attachment, Science 329, 550-553 (2010)

10. F. X. Redl, K.S. Cho, C. B. Murray, S. O’Brien, Three-Dimensional Binary Superlattices of Magnetic Nanocrystals and Semiconductor Quantum Dots. Nature 423, 968-971 (2003).

11. E. V. Shevchenko, D. V. Talapin, N. A. Kotov, S. O'Brien, C. B. Murray, Structural Diversity in Binary Nanoparticle Superlattices. Nature 439, 55-59 (2006).

12. Z. Li, K. Hur, H. Sai, T. Higuchi, A. Takahara, H. Jinnai, S. M. Gruner, U. Wiesner, Linking Experiment and Theory for Three-Dimensional Networked Binary Metal Nanoparticle -Triblock Terpolymer Superstructures, Nat. Commun. 5, 3247 (2014).

13. T. Wang, J. Zhuang, J. Lynch, O. Chen, Z. Wang, X. Wang, D. Lamontagne, H. Wu, Z. Wang, Y. C. Cao, Self-Assembled Colloidal Superparticles from Nanorods, Science 338, 358-363 (2012).

14. G. Jia, A. Sitt, G. B. Hitin, I. Hadar, Y. Bekenstein, Y. Amit, I. Popov, U. Banin, Couples of Colloidal Semiconductor Nanorods Formed by Self-Limited Assembly, Nat. Mater. 13, 301-307 (2014).

15. M. Fukao, A. Sugawara, A. Shimojima, W. Fan, M. A. Arunagirinathan, M. Tsapatsis, T. Okubo, One-Dimensional Assembly of Silica Nanospheres Mediated by Block Copolymer in Liquid Phase, J. Am. Chem. Soc. 131, 16344-16345 (2009). 
16. S. Zhou, Y. Oda, A. Shimojima, T. Okubo, S. Aoshima, A. SugawaraNarutaki, Ring Assembly of Silica Nanospheres Mediated by Amphiphilic Block Copolymers with Oxyethylene Moieties, Polym. J. 47, 128-135 (2014).

17. T. Yanagisawa, T. Shimizu, K. Kuroda, C. Kato, The Preparation of Alkyltrimethylammonium-Kanemite Complexes and Their Conversion to Microporous Materials. Bull. Chem. Soc. Jpn. 63, 988 (1990).

18. C. T. Kresge, M. E. Leonowicz, W. J. Roth, J. C. Vartuli, J. S. Beck, Ordered Mesoporous Molecular-Sieves Synthesized by A LiquidCrystal Template Mechanism. Nature 359, 710 (1992).

19. C. H. Xiao, N. Fujita, K. Miyasaka, Y. Sakamoto, O. Terasaki, Dodecagonal Tiling in Mesoporous Silica. Nature 487, 349 (2012).

20. T. Suteewong, H. Sai, R. Hovden, D. Muller, M. Bradbury, S. M. Gruner, U. Wiesner, Multicompartment Mesoporous Silica Nanoparticles with Branched Shapes: An Epitaxial Growth Mechanism, Science 340, 337-341 (2013).

21. C. C. M. C. Carcouët, Marcel W. P. Van De Put, B. Mezari, P. C. M. M. Magusin, J. Laven, P. H. H. Bomans, H. Friedrich, A. C. C. Esteves, N. A. J. M. Sommerdijk, Rolf A. T. M. Van Benthem, G. D. With, Nucleation and Growth of Monodisperse Silica Nanoparticles, Nano Lett. 14, 1433-1438 (2014).

22. P. A. Korevaar, S. J. George, A. J. Markvoort, M. M. J. Smulders, P. A. J. Hilbers, A. P. H. J. Schenning, T. F. A. De Greef, E. W. Meijer, Pathway Complexity in Supramolecular Polymerization. Nature, 481, 492-496 (2012).

23. A. H. Gröschel, F. H. Schacher, H. Schmalz, O. V. Borisov, E. B. Zhulina, A. Walther, A. H. Müller, Precise hierarchical self-assembly of multicompartment micelles, Nat. Comms. 3, 710-710 (2012).

24. Z. Yu, F. Tantakitti, T. Yu, L. C. Palmer, G. C. Schatz, S. I. Stupp, Simultaneous Covalent and Noncovalent Hybrid Polymerizations, Science 351, 497-502 (2016).

25. K. Ma, C. Mendoza, M. Hanson, U. Werner-Zwanziger, J. Zwanziger, U. Wiesner, Control of Ultrasmall Sub-10 nm Ligand-Functionalized Fluorescent Core-Shell Silica Nanoparticle Growth in Water, Chem. Mater. 27, 4119-4133 (2015).

26. K. Ma, Y. Gong, T. Aubert, M. K. Turker, T. Kao, P. C. Doerschuk, U. Wiesner, Self-assembly of highly symmetrical, ultrasmall inorganic cages directed by surfactant micelles. Nature 558, 577-580 (2018)

27. K. Ma, D. Zhang, Y. Cong, U. Wiesner, Elucidating the Mechanism of Silica Nanoparticle PEGylation Processes Using Fluorescence Correlation Spectroscopies, Chem. Mater. 28 1537-1545 (2016).

28. R. M. Erb, H. S. Son, B. Samanta, V. M. Rotello, B. B. Yellen, Magnetic assembly of colloidal superstructures with multipole symmetry, Nature 457, 999-1002 (2009)

29. M. Cui, T. Emrick, T. P. Russell, Stabilizing Liquid Drops in Nonequilibrium Shapes by the Interfacial Jamming of Nanoparticles, Science 342, 460-463 (2013)

30. K. Ma, U. Werner-Zwanziger, J. Zwanziger, U. Wiesner, Controlling Growth of Ultrasmall Sub-10 nm Fluorescent Mesoporous Silica Nanoparticles, Chem. Mater. 25, 677-691 (2013).

31. Y. Sun, K. Ma, T. Kao, K. A. Spoth, H. Sai, D. Zhang, L. F. Kourkoutis, V. Elser, U. Wiesner, Formation Pathways of Mesoporous Silica Nanoparticles with Dodecagonal Tiling, Nat Comm. 8, 252 (2017)

32. K. Ma, H. Sai, U. Wiesner, Ultrasmall Sub-10 nm Near-Infrared Fluorescent Mesoporous Silica Nanoparticles, J. Am. Chem. Soc. 134, 13180-13183 (2012).

33. Xiao, C., Fujita, N., Miyasaka, K., Sakamoto, Y. \& Terasaki, O. Dodecagonal tiling in mesoporous silica. Nature 487, 349-353 (2012).

34. D. Zhao, Triblock Copolymer Syntheses of Mesoporous Silica with Periodic 50 to 300 Angstrom Pores, Science 279, 548-552 (1998). 


\section{ASSOCIATED CONTENT}

\section{Supporting Information}

The Supporting Information is available free of charge on the ACS Publications website. The Supporting Information contains a PDF file including the section of materials and methods, references (3537), and Figure S1 to S5.

\section{AUTHOR INFORMATION}

\section{Corresponding Author}

${ }^{*}$ Correspondence to: Ulrich Wiesner, ubw1@ cornell.edu

\section{Present Addresses}

† Present address: Elucida Oncology Inc., New York, New York, 10016

\section{Author Contributions}

K.M. and U.W. designed the experimental work. K.M., Y.C., D.Z., T.A., and M.Z.T. synthesized the silica-based materials and performed TEM characterization, supervised by U.W.. K.A.S. and
K.M performed cryo-EM characterization, supervised by L.F.K.. K.M., E.M., and U.W. discussed the formation mechanism. K.M. and U.W. wrote the manuscript with input of all coauthors.

\section{Notes}

A patent disclosure based on this work has been submitted through Cornell Center for Technology Licensing.

\section{ACKNOWLEDGMENT}

The authors gratefully acknowledge support by the National Cancer Institute of the National Institutes of Health under Award Number U54CA199081. K.A.S. and L.F.K. acknowledge support by the Cornell Center for Materials Research (CCMR) with funding from the NSF MRSEC program (DMR-1719875). This work made use of the CCMR shared facilities, and the Nanobiotechnology Center shared research facilities at Cornell. This project has received funding from the European Union's Horizon 2020 research and innovation programme under the Marie Sklodowska-Curie grant agreement No 702300 